\author{
Mohamed Hamza \\ Tripoli University, mohamed_bhamzalyahoo.com, Tripoli-Libya \\ Abduelmajid Najjar \\ Almergeb University, abduelmajid60@yahoo.com, Tripoli-Libya
}

\begin{tabular}{l|l|l|l}
\hline DOI & http: //dx.doi.org/10.12739/NWSA.2018.13.4.3A0085 \\
\hline ORCID ID & $0000-0003-1806-691$ & - \\
\hline \multicolumn{1}{l}{ CORRESPONDING AUTHOR } & Mohamed Hamza
\end{tabular}

\title{
OPTICAL PARAMETERS OF POLYVINYL ALCOHOL-MOLYBDENUM OXIDE COMPOSITE
} FILMS

\section{ABSTRACT}

Polyvinyl alcohol-molybdenum oxide (PVA-MoO 3$)$ composite films were prepared by solution casting technique. The weight percentages of the oxide in the films were 2.5\% and 5\%. The films were characterized by using a computerized double beam UV-VIS spectrophotometer. Optical parameters of the composite films such as real (n) and imaginary (k) parts of refractive index, optical conductivity and dielectric constant were calculated. Other optical parameters (the oscillator energy $\left(E_{0}\right)$ and dispersion energy $\left(E_{d}\right)$ ) have been also evaluated. The obtained results revealed that doping of $\mathrm{MoO}_{3}$ had a significant effect on the optical properties. The results showed that the increased ratio of the oxide led to an increase in the calculated values of the PVA film parameters and shifted toward high wavelength. The addition of $5.0 \%$ of $\mathrm{MoO}_{3}$ to PVA film raised the refractive index (n) value by 38.5\%. The Results of this study show that the $\mathrm{PVA}-\mathrm{MoO}_{3}$ film can have many applications, especially in the area of antireflective coating and photonic devices.

Keywords: Nanocomposite, Optical Parameters, Polyvinyl Alcohol, Molybdenum Oxide, Refractive Index

\section{INTRODUCTION}

Optical property of polymer composite films has attracted much attention due to their application in many fields such as electronic and optical devices. The studies of optical properties are aimed to achieve better reflection, antireflection, interference and polarization. Polyethylene oxide (PEO), polypropylene oxide (PPO) and polyvinyl alcohol (PVA) represent important classes of polymer electrolytes of polar characteristic. These composite are classified as semicrystalline materials. Presence of $\mathrm{OH}$ groups on the polymer chains leads to the formation of hydrogen bonding and, therefore, influences the polymer physical properties [1]. Incorporation of semiconductor nanoparticle inorganic material between chains of transparent polar polymer can display a significant change in the ultimate properties of the composite and may improve their optical properties. The characteristics of these composites can be manipulated by controlling type, size and shape of the nanoparticles and their method of the preparation [2 and 6]. Transition metal oxides are an interesting group of semiconductor materials which could have technological applications, particularly, in display devices, optical smart windows, electrochromic devices, and gas sensors [7 and 8]. Molybdenum oxide $\left(\mathrm{MOO}_{3}\right)$ is among them, which exhibit tremendous structural, chemical, and optical properties. It is considered as a cryogenic material because it displays electrophotochromic and

How to Cite:

Hamza, M. and Najjar, A., (2018). Optical Parameters of Polyvinyl Alcohol-Molybdenum Oxide Composite Films, Physical Sciences (NWSAPS), 13(4):46-54,

DOI: $10.12739 /$ NWSA.2018.13.4.3A0085. 
gasochromic properties by virtue of which gives the material the potential for the development of electronic display devices [9]. $\mathrm{MoO}_{3}$ film has a several application, as in sensors and lubricants [6], and makes it a candidate for back contact layer in cadmium Telluride solar cells [10]. The films can be prepared using different techniques such as thermal evaporation [11], electrodeposition [12] and chemical vapor deposition [13]. However, the poor mechanical strength of pure $\mathrm{MoO}_{3}$ films may limit it uses in specific applications. Incorporation of $\mathrm{MoO}_{3}$ between polyvinyl alcohol chains could improve the mechanical properties widen the application areas. This work is aimed at the preparation of $\mathrm{PVA}^{-\mathrm{MOO}_{3}}$ films with different compositions using PVA as a host polymer taking advantage of its semicrystallinity, ability to form films and having very important applications [1]. This work, also, focused on the investigation of the optical properties of the prepared $\mathrm{PVA}-\mathrm{MOO}_{3}$ films.

\section{RESEARCH SIGNIFICANCE}

Used in the area of antireflective coating. Used in photonic cedices. Used as UV sensor.

\section{Experimental Methods}

\subsection{Materials}

Polyvinyl alcohol (PVA) was obtained from Riedel-de Haen, (Germany) as a yellowish color nontoxic polymer with a $4-6$ CP viscosity and $4 \%$ moisture content at $20^{\circ} \mathrm{C}$. Molybdenum trioxide $\left(\mathrm{MoO}_{3}\right)$ was obtained from Riedel-de Haen, (Germany), as a light gray powder, with a molecular weight of $143.94 \mathrm{~g} / \mathrm{mol}, 99.5 \%$ purity and was used without further purification. Distilled water was used as the solvent throughout the experimental work.

\subsection{Preparation of Composite Films}

Solution casting technique was implemented in the preparation of the polymer composite film (2.5\% and 5.0\% $\left.\mathrm{MoO}_{3}\right)$. One gram of PVA powder was introduced into a beaker containing $25 \mathrm{ml}$ of distilled water, placed on a hot plate fitted with a magnetic stirrer and heated at $70^{\circ} \mathrm{C}$ to dissolve PVA, Exact weight of $\mathrm{MoO}_{3}$ proportional to the percentage of PVA was added while stirring is continued. The dissolved mixture was then poured into a flat bottom glass container of $9 \mathrm{~cm}$ diameter, placed on a flat surface and left to evaporate the solvent at room temperature. The obtained dry film was extracted from the flat container and stored in polyethylene bags in dark place prior to use in the study. Reference pure PVA film (without $\mathrm{MoO}_{3}$ ) was also prepared by the same procedures. The thickness of each film was measured using micrometer having a precision of $0.01 \mathrm{~mm}$ at different parts and the average thickness is calculated to evaluate the uniformity of thickness.

\subsection{Optical Characterization}

The optical transmittance spectra of PVA and PVA-MoO 3 composite films were recorded at room temperature by scanning the prepared films in the wavelength ranged 200-800 nm by using Perkin Elmer double beam UV-VIS spectrophotometer (Model:Lambda 25). The calculation of reflectance (R) was based on the transmittance (T) and the absorbance (A) of the films by the law of energy conservation [14]:

$$
\mathrm{R}+\mathrm{T}+\mathrm{A}=1
$$

Refractive index is one of the most important properties for an optical material because it is closely related to the electronic polarization of ions and the local field inside materials. The 
refractive index (n) and extinction coefficient (k) of the film are calculated using the following mathematical relationships [15]:

$$
\begin{aligned}
& n=\frac{1+\sqrt{R}}{1-\sqrt{R}} \\
& \mathrm{k}=\frac{\alpha \lambda}{4 \pi}
\end{aligned}
$$

Where $\alpha$ is the absorption coefficient and $\lambda$ is the wavelength of the incident light. The dispersion is an important factor in optical communication and also in designing devices for spectral dispersion [16]. The Wemple and DiDomenico single oscillator models are used to analyze the dispersion of refractive index, which provides the useful parameters regarding the strength of inter-band transitions [17]. The dispersion of refractive index below the inter-band absorption edge according to the Wemple and DiDomenico single oscillator model is calculated [18]:

$$
\mathrm{n}^{2}=1+\frac{\mathrm{E}_{0} \mathrm{E}_{\mathrm{d}}}{\mathrm{E}_{0}^{2}-(\mathrm{h} v)^{2}}
$$

Where $E_{0}$ is the average values of single oscillator energy for electronic transitions, which is usually been considered as an average energy gap and $E_{d}$ is the dispersion-energy, which is a measure of the average strength of inter-band optical transitions. It can be considered as a parameter having very close relation with the chemical bonding [19]. The moments of the imaginary part of the optical spectrum $\mathrm{M}_{-1}$ and $\mathrm{M}_{-3}$ are derived from Eqs. (5) and (6) [20]. The static refractive index $\left(n_{0}\right)$ at a zero photon energy is evaluated from Eq. (7) [19]:

$$
\begin{aligned}
E_{0}^{2} & =\frac{M_{-1}}{M_{-3}} \\
E_{d}^{2} & =\frac{M_{-1}^{3}}{M_{-3}} \\
n_{0}^{2} & =1+\frac{E_{d}}{E_{0}}
\end{aligned}
$$

Complex dielectric function $(\varepsilon)$ is a fundamental intrinsic property of the material which measures the ability of a material to interact with an electric field and become polarized by the field. The real part of the dielectric constant displays the level of slowing down the speed of light through the material and it is related to the stored energy within the medium. However, the imaginary part shows a dielectric material absorbing energy from an electric field due to dipole motion. The knowledge of the real and imaginary parts of the dielectric constant provides information about the loss factor which is the ratio of the imaginary part of the real part of the dielectric constant. The real part $\left(\varepsilon_{1}\right)$ and imaginary part $\left(\varepsilon_{2}\right)$ of the dielectric constant can be estimated using the following mathematical relationship [21]:

$$
\varepsilon=\varepsilon_{1}+\varepsilon_{2}
$$

$$
\text { The real and imaginary parts of the dielectric constant ( } \varepsilon_{1} \text { and }
$$
$\left.\varepsilon_{2}\right)$ are calculated from Eq. (9) and (10) [22]:

$$
\begin{aligned}
& \varepsilon_{1}=n^{2}-k^{2} \\
& \varepsilon_{2}=2 \mathrm{nk}
\end{aligned}
$$

Optical conductivity, which represent the electrical conductivity resulting from the movement of the charge carriers due to alternating electric field of the incident electromagnetic waves was also calculated [23]. The optical conductivity $\sigma_{o p}$ is related to the refractive index (n), light speed (c) and absorption coefficient ( $\alpha$ ) and can be calculated by the following equation [23]:

$$
\sigma_{\mathrm{op}}=\frac{\alpha \mathrm{nc}}{4 \pi}
$$




\section{RESULTS AND DISCUSSION}

The spectra of the produced composite films are presented in Figure 1. It shows that the optical transmittance as a function of wavelength of incident light decreases as the concentration of $\mathrm{MoO}_{3}$ increase. This could be contributed to the addition of the metal oxide, which contains electrons in the $\mathrm{MoO}_{3}$ outer orbitals. The absorbed part of the electromagnetic energy of the incident beams led to electronic transition to higher energy levels and occupying vacant positions of energy bands. It has been shown that the pure polyvinyl alcohol film has higher transmittance due to the nonexistence of free electrons in the molecular structure of the material. However, thebonding and excitation of electrons to the conduction band need photon with high energy [22].

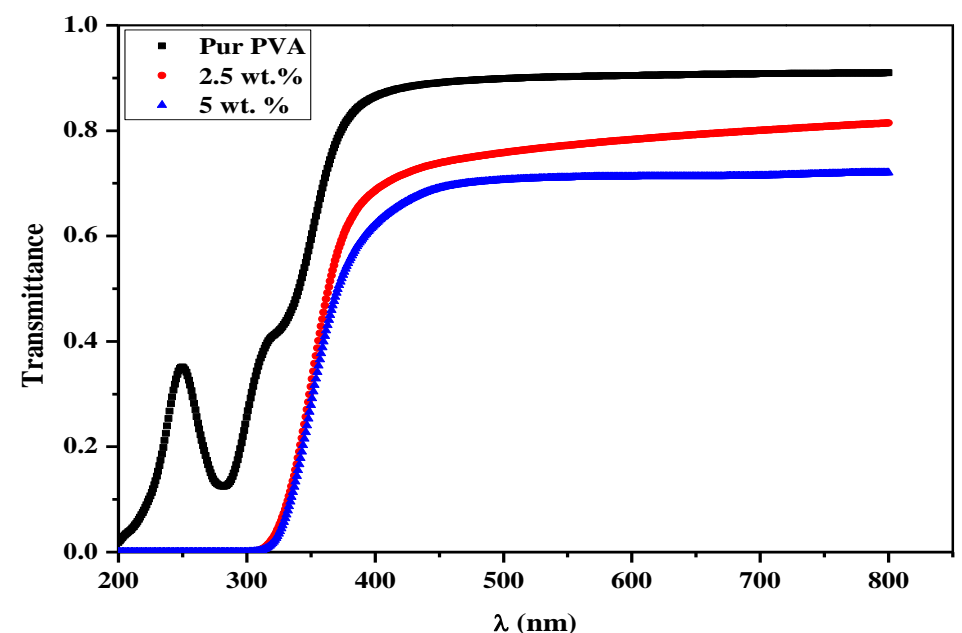

Figure 1. The transmittance spectra for $\mathrm{PVA}-\mathrm{MoO}_{3}$ films as a function of incident wavelength

Figure 2 presents the variations of extinction coefficient (k) with the wavelength for pure PVA and $\mathrm{PVA}-\mathrm{MoO}_{3}$ composite films. It can be seen that the value of $\mathrm{k}$ increases with $\mathrm{MoO}_{3}$ weight percentage increase. The increase of the extinction coefficient for the electrolyte films with $\mathrm{MOO}_{3}$ concentration is due to the increase in the absorption coefficient [24]. This result indicates that the doping of $\mathrm{MoO}_{3}$ tends to change the optical properties of the host polymer.

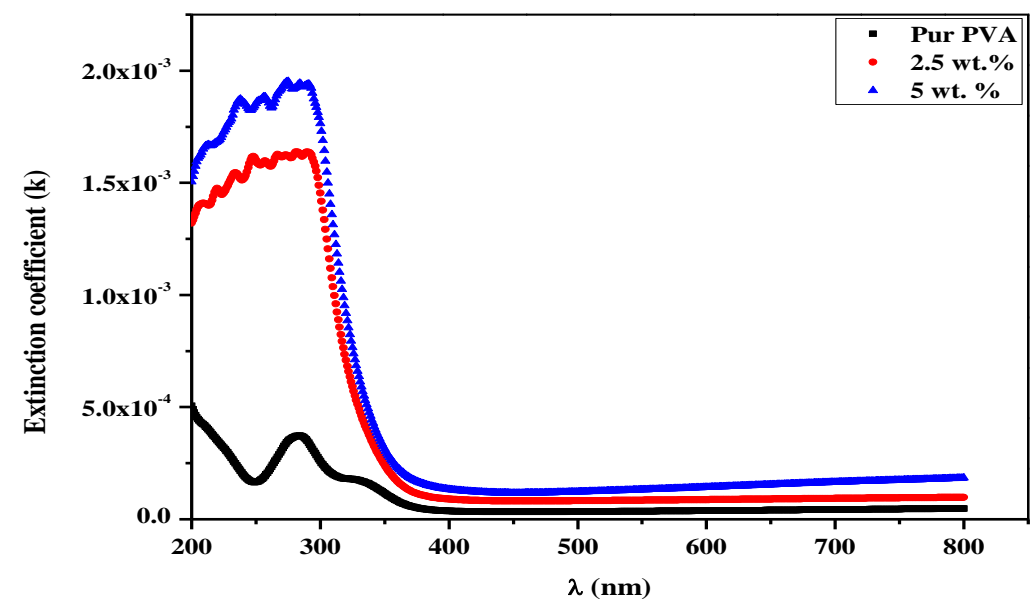

Figure 2. The variation of the extinction coefficient (k) with wavelength of the PVA and PVA- $\mathrm{MOO}_{3}$ composite films 
Hamza, M. and Najjar, A.,

Physical Sciences (NWSAPS), 3A0085, 2018; 13(4): 46-54.

The relationship between the reflectance and the wavelength (Figure 3) shows that the addition of $\mathrm{MoO}_{3}$ (2.5\% and 5.0\%) to PVA shifted the peak position to a higher wavelength. It also shows that the reflectance sharply increases with wavelength and the percentage of $\mathrm{MOO}_{3}$. The finding demonstrated the presence of significant changes in PVA optical properties due to the introduction of $\mathrm{MoO}_{3}$.

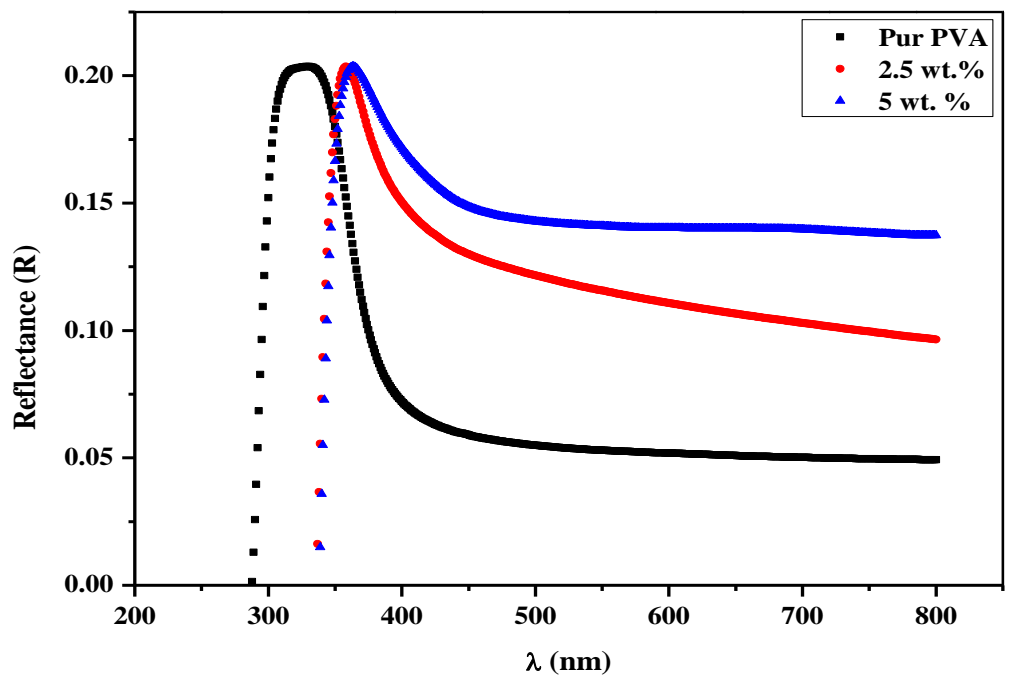

Figure 3. The reflectance of PVA and $\mathrm{PVA}-\mathrm{MoO}_{3}$ films as a function of wavelength

In Figure 4 there is a change in the refractive index of the prepared PVA and $\mathrm{PVA}-\mathrm{MOO}_{3}$ composite films with wavelength. The refractive index decreases with an increase in the wavelength of the incident photon. It is also observed that the value of the refractive index increases with the increase of $\mathrm{MOO}_{3}$ concentration especially at low wavelength region. This type of composite film has a high refractive index which makes it possible to use it in wave guide technology, antireflective coating and photonic devices [25].

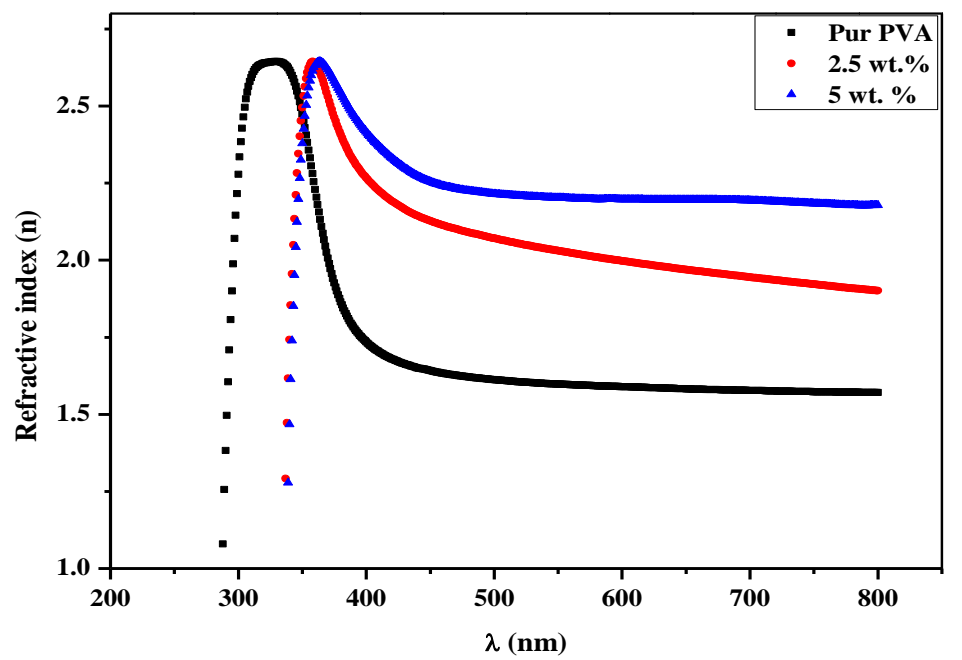

Figure 4. The refraction index of prepared films as a function of wavelength

The values of $E_{0}$ and $E_{d}$ were calculated directly from the slope $\left(-1 /\left(E_{0} E_{d}\right)\right)$ and the intercept $\left(E_{0} / E_{d}\right)$ on the vertical axis of the linear fitted part of $1 /\left(n^{2}-1\right)$ plotted versus $(h v)^{2}$ as shown in Figure 
5. The calculated values of $\mathrm{E}_{0}$ and $\mathrm{E}_{\mathrm{d}}$ for the $\mathrm{PVA}-\mathrm{MoO}_{3}$ composites are listed in Table 1 . The values of $\mathrm{E}_{0}$ and $\mathrm{E}_{\mathrm{d}}$ increase with the increase of $\mathrm{MoO}_{3}$ weight percentage. The increase in the dispersion energy $\left(E_{d}\right)$ indicates the increase in bond strength, which leads to increase in the degree of disorder [19]. The obtained $\mathrm{M}_{-1}$ and $\mathrm{M}_{-3}$ values are also increased with $\mathrm{MoO}_{3}$. The optical moments are related to macroscopic quantities similar to dielectric constants and the effective number of valence electrons in the investigated material [24]. The static refractive index $\left(\mathrm{n}_{0}\right)$ and the static dielectric constant $\left(\varepsilon_{\infty}=\mathrm{n}_{0}{ }^{2}\right)$ were also calculated (Table 1 ). It is clear that the doping of molybdenum oxide within the PVA matrix had a significant effect on the refractive index. The values of $\mathrm{n}_{0}$ and $\varepsilon_{\infty}$ of PVA film are increased when $\mathrm{MoO}_{3}$ is introduced.

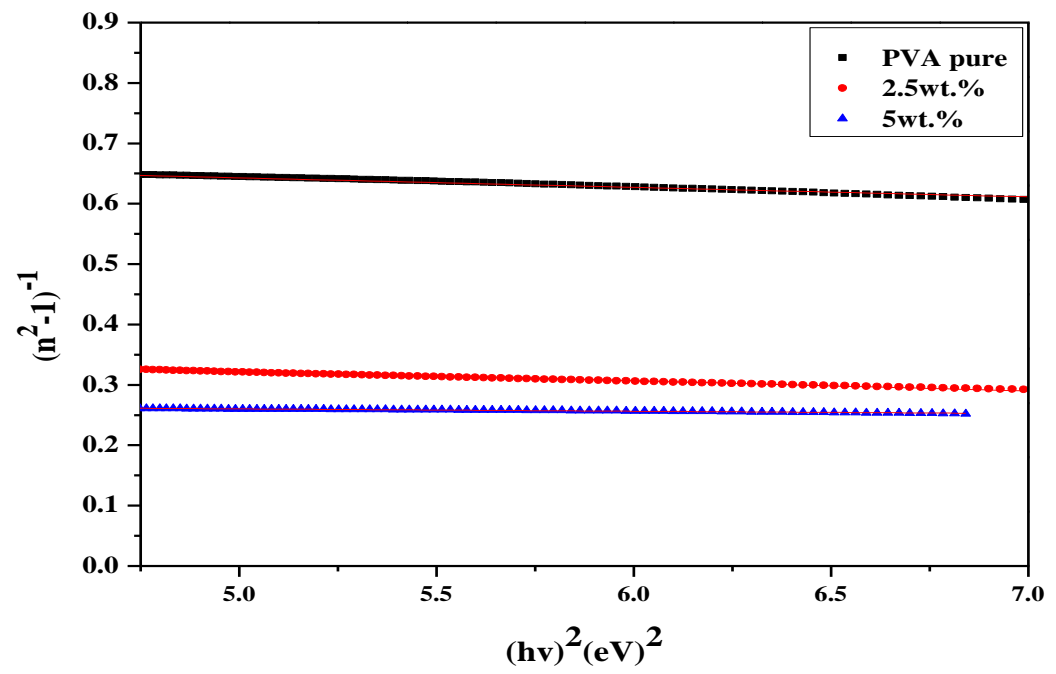

Figure 5. The relationship between $\left(n^{2}-1\right)^{-1}$ and $(h \nu)^{2}$ for PVA and PVA composite contained $2.5 \%$ and $5 \% \mathrm{MoO}_{3}$ films

Table 1. The dispersion parameters and static refractive index of PVA$\mathrm{MoO}_{3}$ composites films

\begin{tabular}{|l|c|c|c|c|c|c|}
\hline $\mathrm{MOO}_{3}$ (wt. 웅 & $\mathrm{E}_{0}(\mathrm{eV})$ & $\mathrm{E}_{d}(\mathrm{eV})$ & $\mathrm{N}_{0}$ & $\epsilon_{\infty}$ & $\mathrm{M}_{-1}$ & $\mathrm{M}_{-3}(\mathrm{eV})^{-2}$ \\
\hline 0 & 6.8027 & 9.4350 & 1.5449 & 2.3869 & 1.3837 & 0.0299 \\
\hline 2.5 & 4.9794 & 12.2757 & 1.8615 & 3.4653 & 2.4646 & 0.0994 \\
\hline 5 & 8.5598 & 30.6619 & 2.1406 & 4.5822 & 3.5829 & 0.0489 \\
\hline
\end{tabular}

The changes in the calculated real and imaginary parts of the dielectric constant of PVA- $\mathrm{MoO}_{3}$ as a function of wavelength are shown in Figures 6 and 7 . The values of both parameters increase with the increase of the weight percentage of $\mathrm{MoO}_{3}$ nanoparticles in the PVA matrix. The variation in the value of $\varepsilon_{1}$ depends mostly on the value of $\mathrm{n}_{2}$ since $\mathrm{k}_{2}$ is so small and the variation of $\varepsilon_{2}$ mainly depends on $\mathrm{k}$ values which is related to the variation of the absorption coefficient. It has been stated that the variations in the real and imaginary parts of dielectric constant with wavelength indicate the interactions between the incident photon and electrons in the composite films. This interaction is wavelength dependent and could be attributed to the orientation of polar groups which depend on the composite types [21]. 
Hamza, M. and Najjar, A., Physical Sciences (NWSAPS), 3A0085, 2018; 13(4): 46-54.

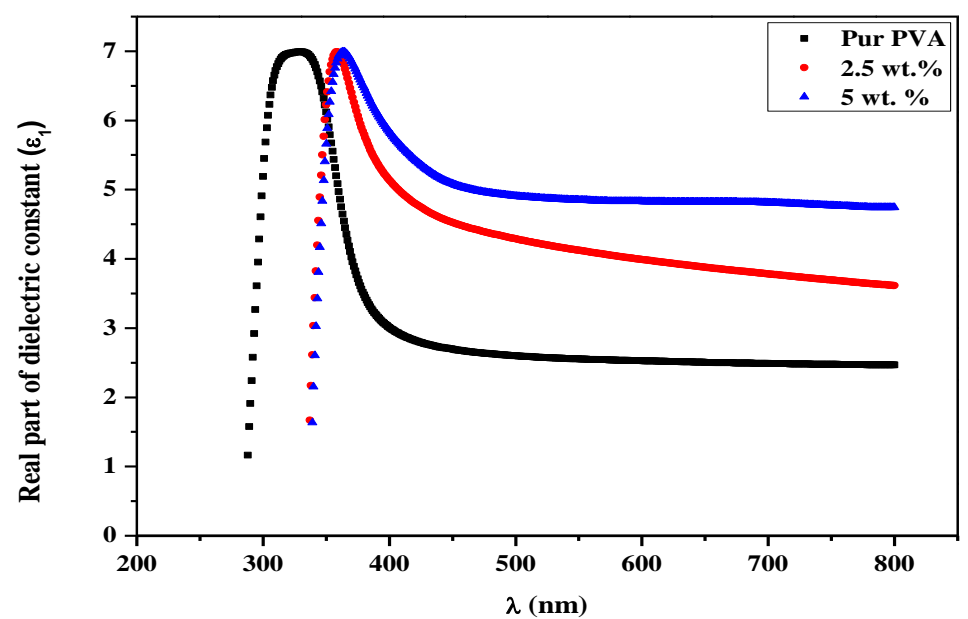

Figure 6. The real dielectric constant for PVA and $\mathrm{PVA}-\mathrm{MoO}_{3} \mathrm{Composite}$ films as a function of incident wavelength

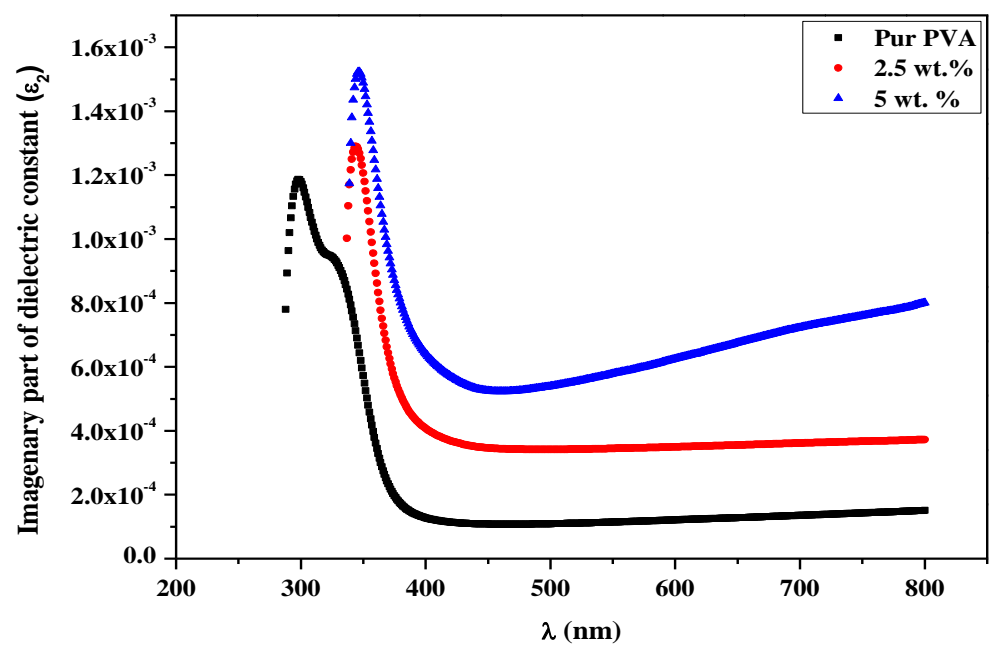

Figure 7. The imaginary dielectric constant of PVA and PVA-MoO composites films as a function of incident wavelength

Figure 8 presents the optical conductivity at different wavelength of pure PVA and $\mathrm{PVA}-\mathrm{MOO}_{3}$ composite films. It is very clear that the addition of $\mathrm{MoO}_{3}$ to PVA matrix has affected the optical conductivity. The peak of PVA film shifts to higher wavelength and optical conductivity. There is an increase in the optical conductivity with the increase of $\mathrm{MoO}_{3}$ weight percentage. This increase can be attributed to the creation of new energy levels in the band gap which facilitate the crossing of electrons from the valence band to these local levels, then to the conduction band and, consequently, the band gap decreased and the conductivity increased [23]. 


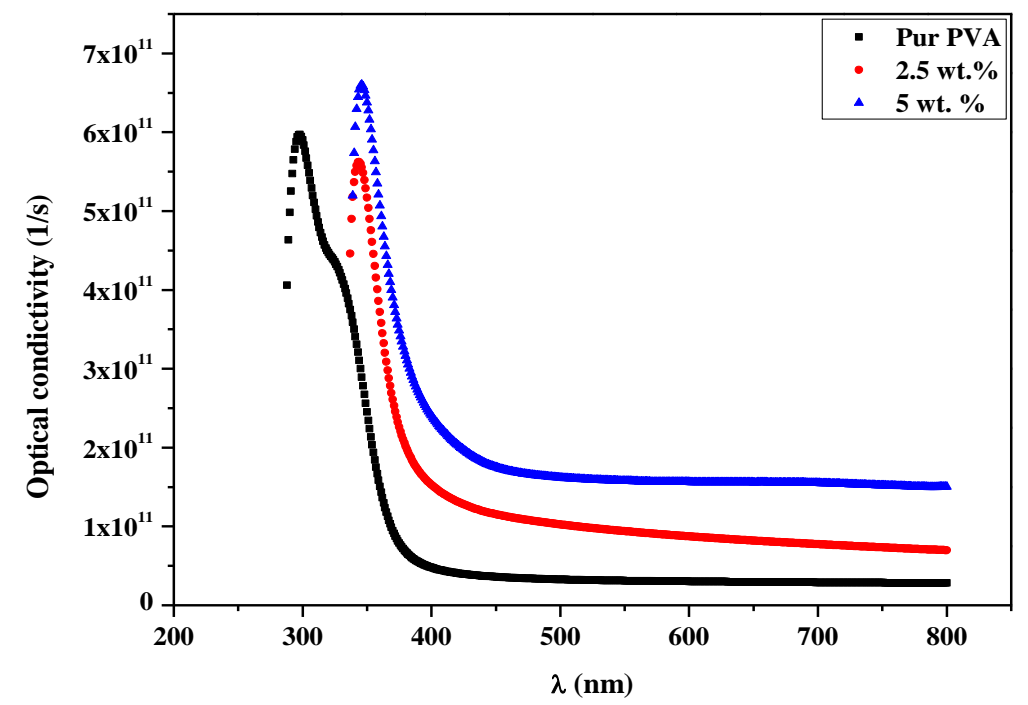

Figure 8. The optical conductivity of the prepared films as a function of incident wavelength

\section{CONCLUSION}

Polyvinyl alcohol and polyvinyl alcohol-Molybdenum oxide composite films are prepared by solution casting method. The optical properties of the produced composite films are studied. Group of parameters which characterize their optical properties included refractive index, extinction coefficient, optical conductivity, dielectric constant, oscillator energy $\left(E_{0}\right)$ and dispersion energy $\left(E_{d}\right)$ are investigated. The optical property of PVA film has been affected by the incorporation of $\mathrm{MoO}_{3}$ within the polymer matrix. The transmittance decreased when the weight percentage of $\mathrm{MoO}_{3}$ increased. The other optical parameters were also increased when $\mathrm{MoO}_{3}$ was introduced. These results conclude that the films we prepared can be used in many applications including antireflective coating and photonic devices.

\section{REFERENCES}

[1] Angham, G.H., Farhan, L., Ahmed, H., Hussein, H., Abbas, I.O., Al-Zuheiry Saba, R.S., (2013). Hind. A. Univ. J. Mater. Sci. $1: 52-55$.

[2] Sarychev, K., Bergman, D.J., and Yagil, Y., (1995). Theory of The Optical and Microwave Properties of Metal-Dielectric Films. Physical Review B. 51:5366-5385.

[3] Helmuth, H.J., (2009). Quant. Spectrodc. Ra. 110:787-799.

[4] Yoochan, H., Yong, M.H., Dae, S.Y., and Jaemoon, Y.J., (1012). Nanobiosensors Based on Localized Surface Plasmon Resonance for Biomarker Detection. Journals of Nanomater. 1-13, ID:759830.

[5] Link, S., and El-Sayed, M.A.J., (1999). Physical Chem B 1999, $103,8410-8426$.

[6] Prasad, A.K., Kubinski, D.J., and Gouma, P.I., (2003). Sensor. Actuator. B. 93:25-30.

[7] Granqvist, C.G., (1995). Handbook of Inorganic Electrochromic Materials, 1st ed. Elsevier, Amsterdam, The Netherlands.

[8] Hinokuma, K., Kishimoto, A., and Kudo, T.J., (1994). Electrochem. Soc. 141:876-879.

[9] Scarminio, J. and Lourenc, A., (1997). Gorenstein, A. Thin. Solid. Films. 302:66-70. 
[10] Gretener, C., Perrenoud, J., Kranz, L., Baechler, C., Yoon, S., Romanyuk, Y.E., Buecheler, S., and Tiwari, A.N., (2013). Thin. Solid. Films. 535:193-197.

[11] Tarsame, S.S. and Reddy, G.B.J., (2005). Appl. Phys. 98:1-9, ID: 026104 .

[12] Patil, R.S., Uplane, M.D., and Patil, P.S., (2006). Structural and Optical Properties of Electrodeposited Molybdenum Oxide Thin Films, Appl. Surf. Sci. 252:8050-8056, Elsivier.

[13] Itoh, T., Matsubara, I., Shin, W., Izu, N., and Nishibori, M., (2008). Preparation of Layered Organic-Inorganic Nanohybrid Thin Films of Molybdenum Trioxide with Polyaniline Derivatives for Aldehyde Gases Sensors of Several Tens PPB Level, Sensor and Actuators B:Chemical, 128:512-520, Elsivier.

[14] Seham, H.S., Esraa, A.A., Enas Y.A., and Shaimaa, A.A., (2016). Effect of Gamma Irradiation on The optical Properties of (PVA: CuCl2) Films, Iraqi Academic Scientific Journals, 57:1968-1974.

[15] Omed, G.A., Bakhtyar, K.A., and Sarkawt, A.H.J., (2013). Chem. Mater. Res. 3:84-90, Elsivier.

[16] Elimat, Z.M., Zihlif, A.M., and Ragosta, G., (2010). Physica B. 405:3756-3760, Elsivier.

[17] Moret, M.P., Devillers, M.A.C., Worhoff, K., and Larsen, P.K.J., (2002). Appl. Phys. 92:468-47, Elsivier.

[18] Wemple, S.H. and DiDomenico. M., (1971). Phys. Rev. B3, 13381351, Elsivier.

[19] Omed, G.A., Yahya, A.K.S., and Salwan, A.S., (2015). Phys. Mater. Chem. 3:18-24, Elsivier.

[20] Wasan, A.A., Mohammed, T.A., and Tagreed, K.A., (2011). The MR affect on Optical Properties for Poly (Vinyl alcohol) Films. Baghdad Science Journal, 8:543-550.

[21] Shehap, A.M. and Dana, S.A., (2016). Int. J. Nanoelectronics and Materials. 9:17-36.

[22] Ghaleb, A.W.A., Hussein, N.N., Ahmed, B., Rafia, T., (2012). Brit. J. Sci. 4:117-124.

[23] Mansour, A.F., Mansour, S.F., and Abdo, M.A.J., (2015). Improvement Structural and Optical Properties of ZnO/PVA Nanocomposites, Jounal of Applied Physics, 7:60-69.

[24] Omed, G.A., Dlear, R.S., Sherzad, A.T., (2015). The Optical Characterization of Polyvinyl Alcohol: Cobalt Nitrate Solid Polymer Electrolzte Films. Advenced Materials Letters. 6:153157 .

[25] Alias, A.N., Zabidi, Z.M., Ali, A.M.M., Harun, M.K., and Yahya, M.Z.A., (2013). Int. J.Appl. Sci. Techn. 3:11-38, Wiley. 